\title{
Building a narrative based Requirements Engineering mediation model
}

\author{
Nan $\mathrm{Ma}^{\mathrm{a}}$, Tracy Hall ${ }^{\mathrm{b}}$, Trevor Barker $\underline{\underline{a}}$ \\ ${ }^{a}$ School of Computer Science, University of Hertfordshire, \\ College Lane, Hatfield A10 9AB, UK \\ \{N.ma, T.1.barker\}@herts.ac.uk \\ ${ }^{\mathrm{b}}$ Department of Information Systems \& Computing, Brunel University, \\ Uxbridge, Middlesex UB8 3P \\ Tracy.Hall@herts.ac.uk
}

\begin{abstract}
This paper presents a narrative-based Requirements Engineering (RE) mediation model to help RE practitioners to effectively identify, define, and resolve conflicts of interest, goals, and requirements. With SPI community, there is a common belief that social, human, and organizational issues significantly impact on the effectiveness of the software process improvement in general and requirements engineering process in particularly. Conflicts among different stakeholders are an important human and social issue that needs more research attention in the SPI and RE community. By drawing on the conflict resolution literature and IS literature, we argue that conflict resolution in $\mathrm{RE}$ is a mediated process, in which a requirements engineer can act as a mediator among different stakeholders. To address socio-psychological aspect of the conflict in RE and SPI, Winslade and Monk (2000)'s narrative mediation model is introduced, justified, and translated into the context of RE.
\end{abstract}

Keywords: Conflict, Method Tailoring, Narrative Mediation, Conflict Resolution, Requirements Negotiation

\section{Introduction}

In this paper we present a narrative-based Requirements Engineering Mediation Model (NREMM). Conflict is a common phenomenon in everyday life [1]. It also has been recognized as an inevitable part of the RE process, as RE is both a social and technical process involving extensive interactions among different stakeholders (e.g. customers, users, developers and testers) from different backgrounds and with different individual and organizational goals [2]. However, in the current RE literature, conflict is consistently considered as a technical issue that may lead to inconsistency in the requirements specification (e.g. [3] [4] [5] [6] [7]). Much work in this area focuses on presenting technical methods or techniques for modelling, analyzing, and managing conflict or inconsistency e.g. KAOS [5], Problem Frames [6] and I* [7] or tools for automating conflict identification and resolution e.g. Oz [8], Synoptic [3], or prompting groupware systems for remote negotiation e.g. Win-Win [9]. Little attention is given to the socio-psychological aspect of the conflict. Furthermore, the term "requirements negotiation" is prevalent in the RE literature where the resolution of 
conflict in RE is considered as a purely negotiation-based process (e.g. [3] [4] [8] [10] [11]) in which a requirements engineer acts as a representative of a developer site and negotiates with users.

This paper adopts a complementary viewpoint and differentiates itself from previous work by recognizing conflict as a social, human, and organizational issue. We adopt Barki and Hartwick's definition of conflict as "a phenomenon that occurs between interdependent parties as they experience negative emotional reactions to perceived disagreements and interference with the attainment of their goals. [12]" Furthermore, we also view the process of resolving conflict in RE is a mediated process, in which a requirements engineer acts as a mediator among different stakeholders.

It is often possible to borrow relevant theories from other disciplines to improve RE practice. Resolving the human aspects of conflict and reaching an agreement in RE can thus be sought by applying relevant approaches that have proved successful in the mediation and conflict resolution discipline. In doing this we borrow the original narrative mediation theory from Winslade and Monk [13] and translate it into the context of RE. This paper aims to describe the rational of why we have built such a model, the methodological approach of how we built it, and finally what a narrativebased RE mediation model is.

This paper is organised as follows: Section 2 gives a review of the relevant literature to justify the rational of building the NREMM model. Section 3 provides an overview of the original narrative mediation model, and justifies its applicability to the context of RE. Section 4 presents our methodological approach of translating the original narrative mediation model into the context of RE, and also presents our NREMM model. Finally, section 5 concludes the paper with some plans for future research.

\section{Conflict resolution in $\mathrm{RE}$}

In this section, we argue that conflict resolution in $\mathrm{RE}$ is a mediation process rather than a negotiation process, in which a requirements engineer acts as a mediator to assist different stakeholders from different backgrounds with different individual and organizational goals to resolve conflicts. The fundamental difference between negotiation and mediation is that, negotiations often only involve conflicting parties themselves reaching an agreement. Mediations then involve a third party as a mediator to lead the process and help parties to reach an agreement.

Most of the RE literature argues that the process of resolving conflict is a purely negotiation-based process, in which a requirements engineer acts as a representative of a development site to "negotiate" with a users' site to make trade-offs (e.g. [3] [4] [8] [10] [11]). However, evidence from the IS discipline suggests that conflicting interests and goals are not only between the users' site and the developers' site, but are often between different user groups. For example, Robertson et al. describe a case where the decision to develop a new production management system was predominantly led by manufacturing and production department specialists who decide to invest heavily in a new manufacturing resources planning system (MRP2) 
[14]. However, in this case, stakeholders from other functional departments (e.g. purchasing and marketing) had different ideas about the problems they were facing and did not believe the new MRP2 to be the solution. Eventually the new system failed due to poor management of such conflicting interests and goals between two users groups [14]. This negotiated form of conflict resolution is seriously questioned in the above situation. It is apparent in the above situation that a requirement engineer needs to play a mediator's role to facilitate the two users groups to reach an agreement on requirements. Our field study of $10 \mathrm{RE}$ practitioners also indicates that RE workshops are the most widely used method of requirements elicitation, and he/she is often required to play the role of a mediator in a RE workshop [15].

The facilitative role of a requirements engineer has been documented in the RE literature. However, there are many diverse views on the facilitators' role in the RE literature ([11] [16]). The role of a requirements engineer as a mediator has not been explicitly identified in the previous RE literature. Few techniques, models, and guidelines have been developed to guide a requirements engineer to resolve conflicting viewpoints in RE practice. In the next section, I will provide a brief overview of the original narrative mediation approach and particularly focus on justifying its applicability and importance to RE.

\section{A brief overview of narrative mediation}

The narrative perspective is that people tend to organize their experiences in story form. In narrative mediation, the process of mediation is thus viewed as a story-telling process [13]. It has been recognized as an innovative conflict resolution paradigm that encourages conflicting parties to reach understanding and resolution through a deep understanding of the shared personal and cultural narratives underlying the conflict. In this section, we provide an overview of the original narrative mediation model, and justify it's applicability to the context of RE.

The narrative approach involves a simple and yet profound departure from commonly held assumptions about the conflicts that embroil people [13]. Its underlying assumption is that people live their lives according to stories rather than according to inner drives or interest. It thus privileges stories and the meanings within stories over facts and causes. In the story, people seek to establish coherence and produce lives, careers, relationship, and communalities [13]. Therefore, when they work with others to overcome the divisiveness of a conflict, they will find it "more productive to work with the stories in which the conflict is embedded than to pursue objective reality" [13]. The original narrative mediation model contains three sub-models [13]:

- Engagement. In this phase, the mediator focuses on establishing a relationship and identifying the problems with the conflicting parties. To achieve a workable relational context, the mediator needs to attend to the physical setting in which the mediation is to take place, to the non-verbal behaviour displayed by all parties, and to the relational moves made by the mediators and the parties. In the case of resolving conflicts in RE, we can refer this phase as conflict identification phases. 
- Deconstructing the conflict-saturated story. This phase of the process involves the mediator developing a supportive relationship and listening respectfully to their own stories. The mediator works actively to separate the parties from their conflict-saturated story. The mediator seeks to undermine the certainties on which the conflict feeds and invites the participants to view the plot of the dispute from a different viewpoint. In the case of resolving conflicts in RE, we can refer this phase as conflict definition phase.

- Constructing the alternative story. In this phase, the mediator is occupied with crafting alternative, more preferred story lines with people who were previously captured by a conflict-saturated relationship. This phase thus may lead to a resolution that takes the form of an agreement between parties. In the case of resolving conflicts in RE, we can refer this phase as conflict solution phase.

\subsection{Narrative mediation's applicability to RE}

We justify the applicability of the original narrative mediation to RE based on the following four aspects:

\section{A process-oriented perspective}

Narrative mediation model adopts a process-oriented perspective. As Winslade and Monk [13] state:

"We have deliberately called this approach a process because we think the word process focuses on the dynamic, shifting, and changing elements of mediation rather than on abstraction, facts, or structures. By concentrating on process, the mediator is invited to think about and work with the responses of the conflicting parties rather than follow some static, preconceived plans."

This process-oriented perspective matches particularly well with the process aspect of $\mathrm{RE}$ practice. RE process is a set of activities that should be systematically followed to derive, validate, and maintain a systems requirements document [2]. The RE literature has presented many different process models, which can range from linear or iterative in structure (e.g. [2] [16]).

Although theses models are explicitly defined in the RE literature, the empirical studies have indicated that the systematic and incremental RE models presented in the $\mathrm{RE}$ literature do not really reflect the reality of RE process in real practice. For example, Hofmann et al., indicate that most companies regard RE as an ad hoc process, with only some using an explicitly defined RE process model or customising a company standard model [17]. Nguyen and Sawtmann also indicate that RE processes do not appear in a systematic, smooth and incremental way, but are "opportunistic, with sporadic simplification and restructuring of the requirements models when points of high complexity are reached" [18]. 
One reason for this chaotic and dynamic RE process is due to requirements changes [19]. It is apparent that the business environment in which software is deployed continually changes. Even if the environment is constant, people's perceptions and understandings are dynamic [20]. As a result, the process of resolving conflicts in RE is a dynamic and complex process. It does not involve discrete stages, and does not follow a tidy sequence of events. Rather, the process moves back and forth in a seemingly dynamic manner when necessary. In this sense, the narrative mediation model which focuses on the dynamic, shifting, and changing elements of mediation seems particularly applicable for the context of RE.

\section{A storytelling process}

Narrative mediation particularly builds on this storytelling metaphor, and provides a mediator with a way of incorporating stories into the resolution of conflict. In narrative mediation, narratives are interactively developed, modified, and contested as parties elaborate portions of their own and each other's conflict stories [13]. This approach thus assumes that conflicts are rooted in conflict-saturated stories that parties have developed through the course of their relationship. As Winslade and Monk state "conflict is likely because people do not have direct access to the truth or the facts about any situation. [13]"

In RE, the way of gathering user requirements fundamentally can be viewed as a storytelling process. New software development methodologies are increasing exploiting to storytelling aspect of RE process (e.g. user stories in XP practice) [21]. Viewing requirements elicitation as a storytelling process not only emphasizes the final outcome - "user stories", but also highlights the importance of verbal communication and interactions between users and developers, which can potentially minimize the ambiguity of requirements specification [22]. In this sense, the original narrative mediation model which builds on the storytelling metaphor seems wellmatched with the fundamental nature of RE elicitation process.

\section{Outsider-in perspective}

The context in which RE takes place is a complex "human activity system"; eliciting and analysing requirements thus can not be performed in isolation from the organizational and social context in which any new system will have to operate [19]. This view stresses a good understanding of the social, political and cultural changes caused by new systems. Moreover, as shown in the Curtis et al.'s classic field study of software engineering process, conflicts result from a wide range of interrelated factors, from change in the organisational setting and business context, to the fact that software will be used by different people with different goals and different backgrounds [23].

In narrative mediation, Winslade and Monk argue an "outsider-in" perspective, which looks at conflict as produced in the socio-culture context, where meanings are contested within the social fabric of community [13]. The narrative mediation approach is based on the idea that people construct conflict from their narrative description of events, and concentrates on developing a relationship that is 
incompatible with conflict and that is built on stories of understanding, respect, and collaboration. The narrative mediation approach recognizes that the mediation context is filled with strong cultural, social, and organizational narratives that form around ethnicity, gender, class, education, financial background, organizational structure and strategies. The narrative mediation approach with an "outsider-in" perspective, which helps mediators and their conflicting parties make sense of the complex social contexts that produce conflicts is thus applicable for the social and organizational aspects of RE.

\section{NREMM}

In this section, we present our NREMM model. The first part of this section explains our models translation approach. Although many existing RE studies present their novel methods or models by borrowing and translating theories from the other disciplines, there is very little in the RE literature that directly and explicitly explains their methodological approach of how their model is systematically and rigorously borrowed and translated. We believe that providing such a methodological approach will benefit further researchers who also seek to translate relevant theories from other disciplines to improve RE practice. However, here we only briefly present our methodological approach and NREMM model. For the detail, please refer to [15].

\subsection{Model Translation Method}

To ensure a rigorous and systematic model translation process, I follow three translation activities (See figure-1):

1. Activity-1: In the first activity, each element of the original narrative mediation model (defined as Model version-V0) is mapped onto the context of RE according to its relevance to the RE literature. This means that all irrelevant elements will be removed from the original model. The outcome of this activity is model version V1, which will retain the structure of the original model but only contain elements relevant to RE. To give a reasonable and subjective assessment of each element's relevance of RE, a scoring scheme was developed and used. A Cohen's Kappa measure of interrater reliability has been carried out, and indicates an acceptable level of agreements $(0.68)$ between two individual raters.

2. Activity-2: A RE specialised mediation model essentially requires the integration of contemporary specialised RE techniques. In the second activity, model version V1 thus will be improved by adding specific RE techniques. The outcome of this activity will be defined as model version V2, which contains specific RE techniques from the RE literature.

3. Activity-3: The original mediation model itself contains a certain degree of overlap. Activity 3 will re-structure the model version V2. 




Figure-1: Three activities of model translation

\subsection{NREMM}

As mentioned in section 3, the original model contains three sub-models, which are also translated into the context of RE: conflict identification (See figure-2), conflict definition (see figure-3), and conflict resolution (see fugirue-4).

\subsubsection{Sub-model-A: conflict identification}

The aim of this phase is to establish a workable relationship with the conflicting parties and initially identify conflict between them. The major activities in this phase include selecting meeting settings, relationship practice, dialogical practice, and stakeholders modelling. The new model below retains majority elements of relational practice, and dialogical practice from the original model, and is complemented by the feature of stakeholder modelling and preparing an RE meeting setting.

\section{Selecting RE meeting setting}

Mediation is a meeting based activity. It is important to ensure a RE meeting take place in the right place, with the group of right stakeholders, and with the facilitation of right artefacts. Therefore, selecting meeting setting in RE focuses on the meeting layout and the use of artefacts. In this research, good practice guidelines (e.g. [16]; [24]) from the existing RE literature are integrated with the original model. 




\section{Stakeholder modelling}

Figure-2: A model of conflict identification

Identifying and involving the right stakeholders is of paramount importance in RE. In particular, stories in RE are interactively written through the collaborations between different stakeholders. Consequently, it is essential to identify the right stakeholder's role and personas prior to listening to his/her conflict story. The disciplines of usercentred design and interaction design provide the theories and techniques for identifying and modelling stakeholders as an initial step towards a successful RE mediation meeting. In this research, we will follow Constantinue and Lockwood's recommended practice to identify and model a useful set of stakeholder roles [25].

\section{Dialogical practice}

Dialogical practice provides a set of questioning and listening technique to develop a dialogue between parties. The key part of dialogical practice in this sub-model is about inviting and listening to the telling of their conflict stories. Narrative mediation requires the mediator should be more interested in learning the story from which the person is operating, not just with the story the parties are telling. The mediator should learn and listen to people as experts on their own lives. Winslade and Monk [13:140] introduce discursive listening techniques and defined it as:

"Careful listening involves hearing not just what has happened but also what necessary constructs are at work in this particular account to make sense of what has happened. This is what we call discursive listening, or listening to the discourses at work in a particular account and to the position calls that are issued within each discourse."

The discursive listening aims to hear the stories as a version or construction of events rather than a set of facts. It does not merely listen for a definable problem, which is some facts that form the basis of the conflict, or the underlying interests of the parties 
that are being expressed in the conflict. Most importantly, discursive listening involves learning and listening for the intersection of narrative in a discursive context.

\section{Relationship practice}

Mediation is a cooperative practice in which the parties to the conflict are viewed as partners in mediation. Thus, at the very beginning, narrative mediation is very much about creating a relational climate. To achieve this relational climate, the original narrative mediation model recommends that a mediator should "show respect to the parties involved, value their personhood, and invite collaborative conversation" Winslade and Monk [13:120]. In the case of RE, it is apparent all these good practices should also be followed by a requirements engineer.

\subsubsection{Sub-model-B: conflict definition (figure-3)}

The aim of this sub-model of mediation is to gain an accurate understanding of conflict. The original narrative mediation model refer to this phase as "deconstructive" in that it gently seeks to undermine the certainties on which the conflict feeds. The sub-model-B thus retains the two elements from the original narrative mediation model: dialogical practice and relationship practice. In addition, the sub-model-B is complemented by adding a new activity: writing a good story.

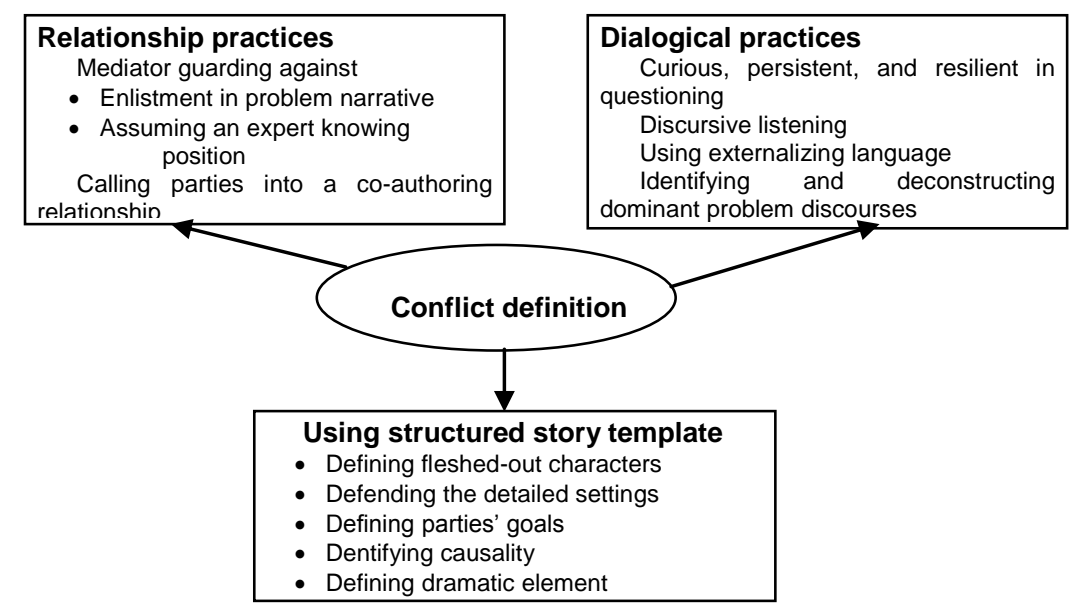

Dialogical practice

Figure-3: Conflict definition

In this phase, the mediator needs to ask questions that will open up space for reconsideration of the conflict story and eventually separate the people from the conflict. Developing an externalizing conversation and questioning curiously play important roles to achieve this. Careful inquiry into the meanings of the elements of the stories that the parties tell seeks to avoid taking any particular meaning for granted. Curious inquiry sometimes needs to be pursued persistently for its best effect. For example, if a developer team speaks about misunderstanding a user's 
interpretation on software requirements as a result of what has happened in a conflicting situation, it might be productive to inquire about the word "misunderstanding" and what it means rather than assume we know what is being referred to. Using this type of questioning technique can break up our sense of certainty that we know all that can be known about what we mean, or even more dangerously, that we know what someone else means[13].

Continuing with above example, we now look how externalizing conversation might be used in the conflicts situation between a user group and a developing team. The mediator might look for some description of the conflict that includes both parties' perspectives. Such a description might need to include notions like betrayal or interference. It might even be called simply the argument. In this case, such a description can be viewed as misunderstanding between users and developers. Then the mediator might speak about the misunderstanding as the cause of two parties' problem, rather than speaking about two parties as the cause of the argument. Such linguistic play, done skillfully, might lead to a new perspective on the conflict, and eventually shifts focuses away from personalities, or blame, and focuses attention on the problematic features of the conflict itself.

\section{Relationship practice}

In this phase of mediation, the relationship established with the parties in the previous preparation phase needs to be continued. In fact, the mediation can proceed only if the mediator is able to continue to demonstrate respect and compassion to the parties. The mediator thus should be "encouraging, affirming trust, having courage to engage with the fullness of the story, and showing impact of conflict story on mediator" [13:80].

\section{Writing a good story}

The original model aims to undermine the fundamental causes of conflicts by adopting unique linguistic techniques such as discursive listening, curiosity questioning, and externalization conversation. The original model strongly emphasises the importance of verbal communication, but overlooks the importance of writing a good story document. The elements added on this activity are adopted from the fields of social science in which the concept and theory of narrative first emerged [26]. Those works recommend the basic practice on writing a good story such as using structured story template, defining fleshed-out characters, defending the detailed settings, defining parties' goals, identifying causality, and defining dramatic element [26].

\subsubsection{Sub-model-C: conflict solution (See figure-4)}

Once the relational issues are addressed in a positive way and the conflict itself is clearly defined, traditional problem-solving based mediation approach can become effectively in this phase. In this sense, a mediator then can begin to invent solutions. The original model asks the mediator to invite parties to identify with their preferred alternative to the conflicting relationship. In the context of RE, this can be understood as the requirements engineer inviting the conflicting stakeholders to propose their 
preferred solutions as the alternatives for the conflicting situation. As a result, this phase will lead to a solution that takes the form of an agreement. The sub-model-C (see figure-4) retains two activities from the original model: dialogical practice and relationship practice. In addition, to help parties reach a fairly objective decision, a semi-quantitative RE prioritization technique is integrated with the original model.



Figure-4: conflict resolution

\section{Relationship practice and dialogical practice}

Although relationship practice and dialogical practice is consistently recognised as two most important parts in the previous two phases of narrative mediation, in this phase of narrative mediation they may not play a most important role comparing with the newly added activity: RE prioritization. This is because that the primary focus of the previous two phases is on identifying and defining conflict. It is inevitable to involve a great deal of verbal communications and relationship practice. However, this phase of narrative mediation focuses on inventing resolution to conflict. It is a problem-solving process, which focuses more on brainstorming, selecting, and evaluating possible solutions. This does not imply that the relational and dialogical practice will be removed from this phase. Instead, all good practices recommended by the original model will be continually retained, but, are considered as less important than RE prioritization.

\section{RE prioritization}

$\mathrm{RE}$ prioritization is widely used to determine the relative necessary of the requirements [27]. Whereas all requirements are mandatory, some are more critical than others. Davis [28] points outs that it particularly aims to resolve conflicts when customer expectations are high, timelines are short, and resources are limited. Indeed, conflicts more likely emerge from those situations. As people naturally have their 
own interests at heart and they aren't always willing to compromise their needs for someone else's benefit. In the context of conflict resolution in RE, RE prioritization can be used to help a mediator to evaluate their preferred solutions and eventually make a win-win decision. In this paper, we will use a semi-quantitative spreadsheet technique based on prioritization of solutions' Value, Cost, and Risk, which is developed by Weigers [29] and described in the figure- 4 .

\section{Conclusion and future work}

This paper presents a RE specialised narrative mediation model. We examined the importance of conflict resolution in $\mathrm{RE}$ and argued that the fundamental nature of conflict resolution in RE is a mediation process. Winslade and Monk (2000)'s narrative mediation model is described, justified, and translated into the context of RE. In the future, the newly developed model is about to be tested in the real-world contexts.

\section{References}

1. Pruitt, D. G., \& Kim, S. H., Social conflict: Escalation, stalemate, and settlement (3rd ed.). New York: McGraw-Hill. 2004

2. Sommerville I. and Sawyer P. Requirements Engineering: A Good Practice Guide, Wiley, 1997,

3. Easterbrook S. M. "Resolving Requirements Conflicts with Computer-Supported Negotiation". In M. Jirotka \& J. Goguen (eds) Requirements Engineering: Social and Technical Issues, Pp41-65. London: Academic Press. 1996

4. Nuseibeh B., To Be And Not To Be: On Managing Inconsistency in Software Development, Proceedings of 8th International Workshop on Software Specification and Design (IWSSD-8), pp164-169, Scloss Velen, Germany, 22-23 March 1996, IEEE CS Press.

5. Van Lamsweerde, A. Requirements Engineering in the Year 00: A Research perspective, Invited Paper for ICSE'2000 - 22nd International Conference on Software Engineering, Limerick, ACM Press, June 2000.

6. Jackson M. "Problem Frames: Analysing and Structuring Software Development Problems" Addison-Wesley Longman Publishing Co., Inc. 2001

7. Yu, E. and Mylopoulos, J. Why Goal-Oriented Requirements Engineering, Fourth International Workshop on Requirements Engineering: Foundation for Software Quality (REFSQ'98), E. Dubois, A. Opdahl and K. Pohl (ed.), Pisa, Italy, 1998

8. Robinson, W.N. Negotiation Behaviour During Multiple Agent Specification: A Need for Automated Conflict Resolution, Proceedings of 12th International Conference on Software Engineering (ICSE-12), 268-276, Nice, France, IEEE Computer Society Press, March 1990

9. Boehm, B. Grünbacher P. and Briggs R. Developing Groupware for Requirements Negotiation: Lessons Learned, IEEE Software, 18(3), 2001

10. Damian D.E., Shaw, M.L.G., Gaines, B.R. and Zowghi, D. A multi-disciplinary approach to the study of distributed requirements negotiations, Proc. of the 5th Australian Workshop on Requirements Engineering, 91-100, December 8-9, Brisbane, Australia, 2000

11. Damian, D.E. A research methodology in the study of requirements negotiations in geographically distributed software system, Proceedings of 11 the IEEE International Requirements Engineering Conference, 2003 
12. Barki $\mathrm{H}$, Hartwick J, Interpersonal conflict and its management in information system development, MIS Quarterly Vol. 25 No.2, pp195-228, June 2001

13. Winslade J. and Monk G. , Narrative Mediation: A New Approach to Conflict Resolution" Jossey-Bass, 2000

14. Robertson M, Swan J, Newell S. "The role of networks in the diffusion of technological innovation". Journal of manage studies; 33(3):335-26, 1996

15. Ma N., Hall T., Barker T., "Using an expert panel to validate a Requirements Engineering Mediation Model" a paper submitted on EASE 2008 conference, 26, June, Italy, 2008

16. Macaulay L. (1996), Requirements engineering. Springer-Verlag Series on Applied Computing, London, UK

17. Hoffmann, O., Cropley, D., Cropley, A., Nguyen, L., Swatman, P. (2005) Creativity, Requirements, and Perspectives, Australian Journal of Information Systems13(1), pp159 $-175$

18. Nguyen, L and Swatman, P., (2006). Promoting and Supporting Requirements Engineering Creativity (chapter 10) In Rationale Management in Software Engineering, edited by A. H. Dutoit, R. McCall, I. Mistrik, and B. Paech, Springer-Verlag.

19. Nuseibeh B. and Easterbrook S., Requirements Engineering: A Roadmap , Proceedings of International Conference on Software Engineering (ICSE-2000), 4-11 June 2000, Limerick, Ireland

20. Sommerville Ian Integrated Requirements Engineering: A Tutorial. IEEE Software 22(1): 16-23, 2005

21. Beck, K. Extreme Programming Explained: Embrace Change - Addison Wesley - 2000.

22. Cohn, Mike "User Stories Applied: For Agile Software Development" Boston, AddisonWesley Signature Series 2004

23. Curtis B., Krasner H. and Iscoe N., A field study of the software design process for large systems, Communications of the ACM 31 (1988) (11), pp. 1268-1287

24. Maiden N., Bright B.P.: Recurrent communication patterns in requirements engineering meetings. WETICE 1996: 208-213

25. Constantine, L. L., and Lockwood, L. A. D. Software for Use: A Practical Guide to the Essential Models and Methods of Usage-Centered Design. Reading, MA: AddisonWesley, 1999

26. Burroway, J. Writing Fiction: A Guide to Narrative Craft. Addison-Wesley. (1999)

27. Fellows L. and Hooks I., "A Case for Priority Classifying Requirements," Eighth Annual International Symposium on Systems Engineering, Seattle, Washington: International Council on Systems Engineering, 1998.

28. Davis (2003) A., "The Art of Requirements Triage," Computer, Vol. 36, No. 3, March 2003, pp. 42-49

29. Wiegers Karl E., (1999) First Things First: Prioritizing Requirements, Software Development, Vol. 7, no. 9 